\title{
Simultaneous State and Parameter Estimation for Physics-Based Tracking of Heart Surface Motion
}

\author{
Evgeniya Bogatyrenko and Uwe D. Hanebeck
}

\begin{abstract}
Most existing approaches for tracking of the beating heart motion assume known cardiac kinematics and material parameters. However, these assumptions are not realistic for application in beating heart surgery. In this paper, a novel probabilistic tracking approach based on a physical model of the heart surface is presented. In contrast to existing approaches, the physical information about heart kinematics and material properties is incorporated and considered in an estimation of the heart behavior. An additional advantage is that the time-dependencies and uncertainties of the heart parameters are efficiently handled by exploiting simultaneous state and parameter estimation. Furthermore, by decomposing the state into linear and nonlinear substructures, the computational complexity of the estimation problem is reduced. The experimental results demonstrate the high performance of the method proposed in this paper. The solution of the parameter identification problem allows a personalized physical model and opens up possibilities to apply the physics-based tracking of the heart surface motion in a clinical environment.
\end{abstract}

\section{INTRODUCTION}

Beating heart operations are still a challenging task for surgeons. A robotic surgery system will assist a surgeon during an operation on a beating heart by tracking heart surface motion and synchronizing surgical instruments.

For an application in a robotic surgery system, a variety of tracking approaches exists in related work [1]-[4]. In contrast to other approaches, the physics-based tracking [3], [4] guarantees a physically correct prediction of the heart behavior. This tracking is based on a physical heart model and thus, exploits additional background information about the physical characteristics of the heart. However, in case of an incorrect model or false parameters [5], the physics-based tracking fails.

Therefore, the parameter identification problem is one of the most important issues concerning the estimation and tracking of heart surface motion. The main goal is the estimation of the system parameters from observed measurements, such that the predicted response of the heart model is close to the real heart behavior. A common way is to determine the parameters from preoperative data [6], [7]. Employing the preoperative detected parameters for an intraoperative physical model can lead to instabilities and inaccuracies of physics-based tracking. The reason for this

This work was partially supported by the German Research Foundation (DFG) within the Research Training Group RTG 1126 "Intelligent Surgery - Development of new computer-based methods for the future working environment in visceral surgery".

E. Bogatyrenko and U. D. Hanebeck are with the Intelligent Sensor-Actuator-Systems Laboratory (ISAS), Institute for Anthropomatics, Karlsruhe Institute of Technology (KIT), Karlsruhe, Germany. bogatyrenko@kit.edu, uwe.hanebeck@ieee.org is changing clinical environment, tissue properties and heart dynamics after preoperative planning. Therefore, for beating heart operations, an adjustment of the parameters featured in the intraoperative physical model is necessary for reducing the gap between the predicted response of the model and the real heart behavior. Until now, a personalized heart model for physics-based tracking in a robotic surgery on a beating heart has not been proposed [3], [4]. The main challenge of an intraoperative parameter identification is that the state and parameter estimation leads to a high-dimensional nonlinear estimation problem. Even an originally linear parameter system becomes nonlinear when the system state is augmented with the model parameters. An efficient solution of this problem is a prerequisite for real-time functionality of a robotic surgery system.

The novelty of this paper is the systematic probabilistic approach for physics-based tracking and parameter identification that aims to be applied in intraoperative medical applications. The model and measurement uncertainties are considered. By decomposing the state into linear and nonlinear substructures, the computational complexity of the estimation problem is reduced. A further advantage of this approach is an incorporation of the a-priori known physical constraints in the identification of the model parameters.

The remainder of this paper is structured as follows: Section II gives an overview of the probabilistic physicsbased tracking approach proposed in this paper. In Section III, the system and measurement models for physics-based tracking of heart surface motion are formulated. Section IV considers the initialization of the system model. The system decomposition into linear and nonlinear substructures and simultaneous state and parameter identification procedures are derived in Section V. The performance of the proposed approach is demonstrated by simulations and experiments in Section VI. Finally, the paper closes with a conclusion and an outlook to future work.

\section{SYSTEM OVERVIEW}

As an introduction to our probabilistic physics-based tracking approach, this section will give an overview of existing state and parameter estimation methods. A motivation for their extension and further development will also be given.

For tracking heart surface motion, the heart kinematics should be predicted by means of an estimation approach. For that purpose, the system model should be calibrated by identifying the parameters and stochastic uncertainties of the system. Generally, there exist two methods for the state 


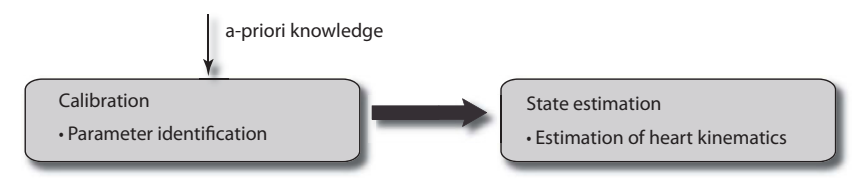

(a) Strict separation of the parameter identification problem from the state estimation.

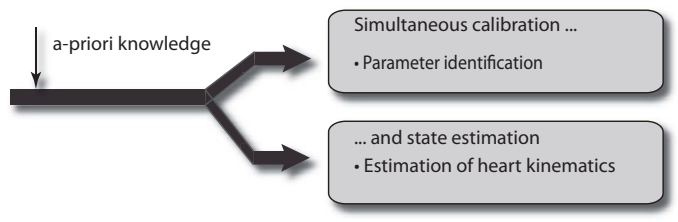

(b) Simultaneous state and parameter estimation.

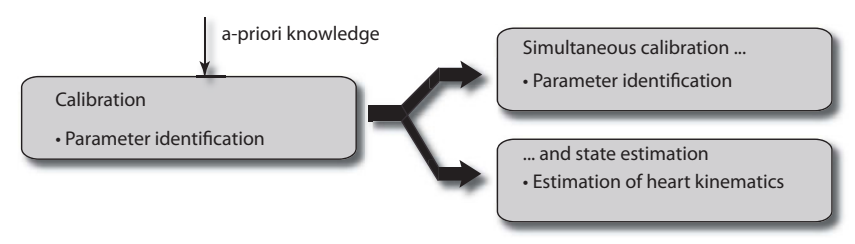

(c) Simultaneous state and parameter estimation with a pre-calibration procedure.

Fig. 1. Methods for the state and parameter estimation regarding physicsbased tracking in a robotic surgery system.

and parameter estimation of a distributed-parameter system (DPS) [8]:

1) strict separation of the parameter identification problem from state estimation and

2) simultaneous state and parameter estimation.

By strictly separating the parameter identification problem from the state estimation, the system is calibrated before estimating the heart kinematics, as shown in Fig. 1(a). The calibration procedure incorporates a-priori knowledge about the physical system, such as physical constraints on model parameters. Then, if the heart surface motion is described by a linear system, by assuming the parameters of the system as known and time-independent, it is sufficient to use the Kalman filter to obtain the best possible estimate for the system state. However, due to inaccuracy of the model calibration or when the heart parameters changes over a cardiac cycle, large estimation errors can arise. The heart surface motion prediction, based on a model with incorrect parameters, will strongly deviates from the true state.

A remedy for this problem is the simultaneous state and parameter estimation, presented in Fig. 1(b). In this case, by considering the state and parameter uncertainties simultaneously, we significantly increase the performance of the prediction and thus, of the physics-based tracking. Moreover, the time-dependence of the parameters can be handled systematically. However, the task of simultaneous state and parameter estimation of a distributed-parameter system, such as the heart, is very challenging. The main reason is that the required spatial resolution leads to large-scale models with a high number of state variables. In addition, an augmentation of the system state with the model parameters leads to a nonlinear estimation problem. As a consequence, the computational cost of the estimation becomes prohibitive. Furthermore, the stability of the estimation is strongly de- pendent on the initial estimates of the physically constrained system parameters.

Therefore, in order to avoid the instabilities of the simultaneous state and parameter estimation due to poor initial estimates, we propose a pre-calibration of the system, as presented in Fig. 1(c). For achieving an efficient simultaneous state and parameter estimation, the large-scale system model describing the heart motion is decomposed into linear and nonlinear substructures. Moreover, by incorporating the physical constraints on the model parameters into the estimation process, we guarantee the convergence of estimates.

\section{PhysiCs-BASED DESCRIPTION OF HEART SURFACE MOTION}

Before the mathematical background of the approach for parameter identification is explained, this section will formulate the system and measurement models for physicsbased tracking of the heart surface motion. For reducing the dimensionality of the heart model, we constrain the tracking to the intervention area. By assuming that the heart surface in this area behaves like a linear elastic physical body with isotropic material structure, the heart dynamics can be approximately characterized by a discrete system equation, or system model [3], [4], [7]

$$
\underline{\boldsymbol{x}}_{k+1}=\mathbf{A}_{k}\left(\underline{\boldsymbol{\theta}}_{k}\right) \underline{\boldsymbol{x}}_{k}+\mathbf{B}_{k}\left(\underline{\boldsymbol{\theta}}_{k}\right)\left(\underline{\hat{u}}_{k}+\underline{\boldsymbol{w}}_{k}\right),
$$

where the vector

$$
\underline{\boldsymbol{x}}_{k}=\left[\underline{\boldsymbol{c}}_{k}^{\mathrm{T}}, \underline{\dot{\boldsymbol{c}}}_{k}^{\mathrm{T}}\right]^{\mathrm{T}} \in \mathbb{R}^{6 N_{x}}
$$

represents the discrete states of the heart movement at sequential time steps $t_{k+1}$ and $t_{k}$. These states describe cardiac kinematics, such as three-dimensional displacement of the heart surface and its velocity on $N_{x}$ points. The error term $\underline{\boldsymbol{w}}_{k} \sim \mathcal{N}\left(0, \mathbf{C}_{k}^{w}\right)$ is a white zero-mean Gaussian noise with covariance $\mathbf{C}_{k}^{w} \in \mathbb{R}^{6 N_{x} \times 6 N_{x}}$. The unknown model parameters influencing the computation of the system matrix $\mathbf{A}_{k} \in \mathbb{R}^{6 N_{x} \times 6 N_{x}}$ and input matrix $\mathbf{B}_{k} \in \mathbb{R}^{6 N_{x} \times N_{u}}$ are denoted by the vector

$$
\underline{\boldsymbol{\theta}}_{k}=\left[\boldsymbol{\rho}_{k}, \boldsymbol{\alpha}_{1 k}, \boldsymbol{\alpha}_{2 k}, \boldsymbol{E}_{k}, \boldsymbol{\nu}_{k}\right]^{\mathrm{T}} .
$$

These parameters describe physical characteristics of the heart surface, such as material density $\boldsymbol{\rho}_{k}$, Rayleigh damping coefficients $\boldsymbol{\alpha}_{1 k}, \boldsymbol{\alpha}_{2 k}$, Young's modulus $\boldsymbol{E}_{k}$, and Poisson's ratio $\boldsymbol{\nu}_{k}$. According to [4], the system input $\underline{\hat{u}}_{k}=\left[\underline{\hat{b}}_{k}^{\mathrm{T}}, \underline{\hat{g}}_{k}^{\mathrm{T}}\right]^{\mathrm{T}}$ contains the known forces $\underline{\hat{b}}_{k}$ and boundary conditions $\underline{\hat{g}}_{k}$.

Assuming that the discrete-time measurements of the heart kinematics are provided by a camera system, the measurement model relates the obtained observations $\underline{\hat{y}}_{k} \in \mathbb{R}^{L}$ at time step $k$ to the state $\underline{x}_{k} \in \mathbb{R}^{6 N_{x}}$ by

$$
\underline{\hat{y}}_{k}=\mathbf{H}_{k} \underline{\boldsymbol{x}}_{k}+\underline{\boldsymbol{v}}_{k},
$$

where $\mathbf{H}_{k} \in \mathbb{R}^{L \times 6 N_{x}}$ represents the measurement matrix [4] and $\underline{\boldsymbol{v}}_{k} \sim \mathcal{N}\left(0, \mathbf{C}_{k}^{v}\right)$ is a white zero-mean Gaussian noise with covariance $\mathbf{C}_{k}^{v} \in \mathbb{R}^{L \times L}$.

As a result, the heart surface motion description is given in a discrete finite-dimensional state-space form, which is 
required for solving estimation problems. For the physicsbased tracking of the heart surface motion, an accurate prediction is essential. The quality of the prediction depends strongly on the model quality and parameters.

\section{Model Calibration}

The calibration procedure presented in this section determines model parameters, so that the predicted response of the deterministic heart model is close to the true heart behavior. The goal of this procedure is to provide initial estimates of the model parameters for simultaneous state and parameter estimation.

The calibration makes use of observations $\underline{\hat{y}}_{k}$ of the heart surface motion over $N$ time steps of an initialization time interval. For fitting the heart model to the measurement data, we apply the weighted least-squares approach by minimizing the cost function [9]

$$
\underline{J}\left(\underline{\theta}_{k}\right)=\frac{1}{2} \sum_{k=1}^{N}\left\|\underline{\hat{y}}_{k}-\underline{y}_{k}\left(\underline{\theta}_{k}\right)\right\|_{\left(\mathbf{Q}_{k}\right)^{-1}}^{2},
$$

where the weighting matrix $\mathbf{Q}_{k} \in \mathbb{R}^{L \times L}$ is assumed to be positive definite with

$$
\|a\|_{\left(\mathbf{Q}_{k}\right)^{-1}}^{2}=a^{\mathrm{T}}\left(\mathbf{Q}_{k}\right)^{-1} a, \forall a \in \mathbb{R}^{L} .
$$

Here, $\underline{y}_{k} \in \mathbb{R}^{L}$ stands for the predicted values corresponding to the parameter vector $\underline{\theta}_{k}$.

In order to ensure the physical meaning of the model parameters, the physical constraints are incorporated into the calibration procedure. By fitting the model to the observations, only the parameters from the bounded intervals are considered.

It is known that since the weighted least-squares approach uses iterative methods for finding a minimum, only local minima are typically found. Therefore, by means of simultaneous state and parameter estimation, the accuracy of the parameter identification can be improved.

\section{Simultaneous State and Parameter Estimation}

The simultaneous state and parameter estimation of the heart surface motion copes with the changes of the heart parameters during a surgical operation. For this purpose, the parameter vector is included as an additional part of the state vector. As a result, the augmented system model is generally high-dimensional and nonlinear in the augmented state. The Bayesian estimation framework allows recursively processing the state of a nonlinear dynamic system by incorporating the observed measurements. Therefore, conventional estimation techniques can be used to estimate the parameter and states simultaneously. An approach for simultaneous state and parameter estimation, to be applied in physics-based tracking of the heart surface motion, is proposed in this section.

The state-space model of the heart surface motion derived in Section III contains a conditionally linear system equation (1). The parameter vector (3) and the input vector are assumed as random processes. Exploiting the decomposition of this equation into linear and nonlinear substructures by means of Rao-Blackwellization [10], we reduce the estimation problem and execute the estimation in an efficient way. For prediction, the Gaussian estimator (GE) introduced in [11], [12] is applied. For efficiency of the estimation, only the nonlinear part is processed by GE in an approximate fashion. Since the measurement equation is linear (4), the Kalman filter measurement update equations can be used.

\section{A. Prediction Step}

For efficiency of the estimation, the augmented state can be decomposed into linear and nonlinear substructures, similar to [8], [12]

$$
\underline{\boldsymbol{z}}_{k}=\left[\begin{array}{l}
\underline{\boldsymbol{x}}^{n} \\
\underline{\boldsymbol{x}}^{l}
\end{array}\right],
$$

where the linear substructure $\underline{\boldsymbol{x}}^{l}=\underline{\boldsymbol{x}}_{k}$ contains the state describing the heart kinematics, which is given in equation (2). The nonlinear substructure $\underline{\boldsymbol{x}}^{n}=\underline{\boldsymbol{\theta}}_{k}$ consists of parameters (3) influencing the computation of the system matrices. The state $\underline{\boldsymbol{z}}_{k}$ is assumed to be Gaussian distributed. The mean and covariance of the estimated state are defined as

$$
\underline{\mu}_{e}^{z}=\left[\begin{array}{c}
\underline{\mu}^{n} \\
\underline{\mu}^{l}
\end{array}\right], \mathbf{C}_{e}^{z, z}=\left[\begin{array}{ll}
\mathbf{C}^{n, n} & \mathbf{C}^{n, l} \\
\mathbf{C}^{l, n} & \mathbf{C}^{l, l}
\end{array}\right] .
$$

Similar to [12], the system equation (1) is formulated as a conditionally linear model in the form

$$
\underline{\boldsymbol{z}}_{k+1}=\underbrace{\left[\begin{array}{l}
\mathbf{P}_{k} \underline{\boldsymbol{\theta}}_{k} \\
\mathbf{B}_{k}\left(\underline{\boldsymbol{\theta}}_{k}\right) \underline{\boldsymbol{u}}_{k}
\end{array}\right]}_{\underline{g}\left(\underline{\boldsymbol{x}}^{n}\right)}+\underbrace{\left[\begin{array}{l}
\mathbf{0} \\
\mathbf{A}_{k}\left(\underline{\boldsymbol{\theta}}_{k}\right)
\end{array}\right]}_{\mathbf{H}\left(\underline{\boldsymbol{x}}^{n}\right)} \underline{\boldsymbol{x}}^{l}+\underline{\boldsymbol{w}}_{k},
$$

where the matrix $\mathbf{P}_{k}$ characterizes the space and timedependence of the parameter vector $\underline{\theta}_{k}$ given in equation (3). This matrix turns to an identity matrix $\mathbf{I}$ if a random walk model of the spatially independent parameters is assumed. The covariance matrix of additive noise process $\underline{\boldsymbol{w}}_{k}$ is defined as

$$
\mathbf{C}_{k}^{w}=\left[\begin{array}{ll}
\mathbf{C}_{k}^{x^{n}} & \mathbf{0} \\
\mathbf{0} & \mathbf{C}_{k}^{x^{l}}
\end{array}\right]
$$

Then, the $M$-dimensional Gaussian density of the nonlinear part of the state $f\left(\underline{\boldsymbol{x}}^{n}\right)$ is approximated with a Dirac mixture density based on the deterministic sampling approach presented in [11]

$$
f\left(\underline{x}^{n}\right) \approx \sum_{i=1}^{L} w \cdot \delta\left(\underline{x}^{n}-\underline{\mu}_{i}^{n}\right)
$$

with $L=M \cdot(D-1)+1$, where $D$ is the number of sampling points with weights $w=\frac{1}{L}$. Furthermore, the first and second moments of the predicted state are calculated according to [12] by

$$
\begin{aligned}
\underline{\mu}_{k+1}^{p} & =w \cdot \sum_{i=1}^{L} \underline{\mu}_{i}^{z}, \\
\mathbf{C}_{k+1}^{p} & =\sum_{i=1}^{L}\left(w \cdot \mathbf{C}_{i}^{z, z}+w_{s} \cdot\left(\underline{\mu}_{i}^{z}-\underline{\mu}^{z}\right)\right. \\
& \left.\cdot\left(\underline{\mu}_{i}^{z}-\underline{\mu}^{z}\right)^{\mathrm{T}}\right)+\mathbf{C}_{k}^{w},
\end{aligned}
$$




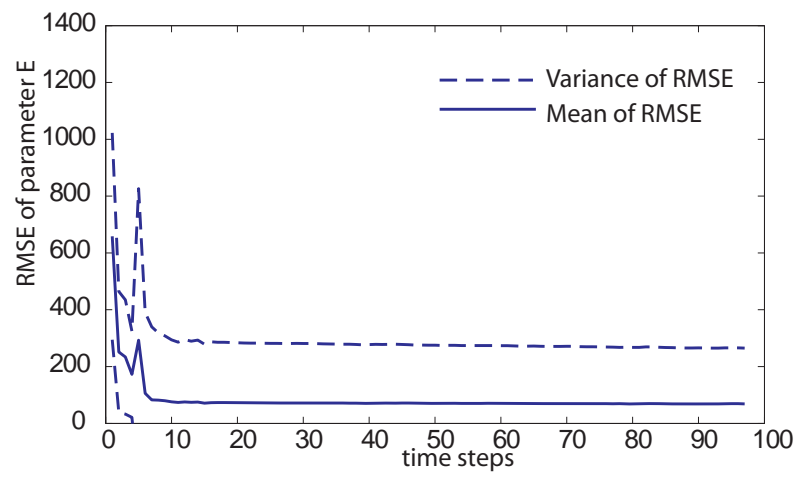

(a) Root-mean-square error (RMSE) of the estimated parameter Young's modulus $E$ compared to the true Young's modulus over 50 simulations for time steps 1 to 100 .

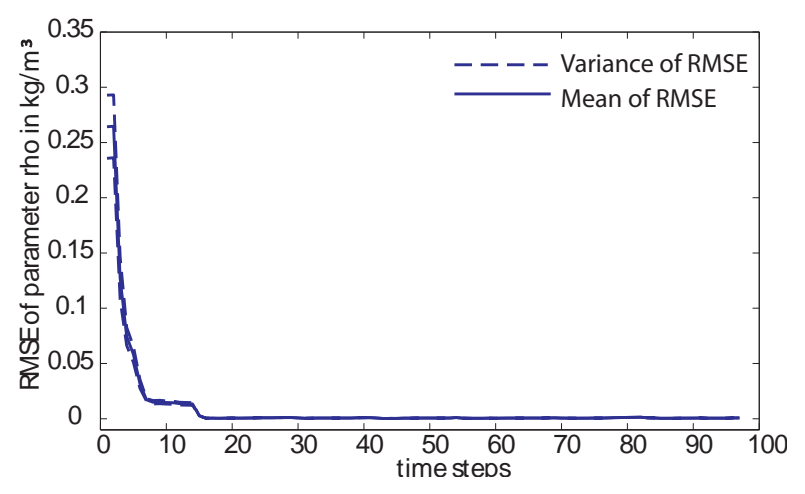

(b) RMSE of the estimated material density $\rho$ compared to the true material density over 50 simulations for time steps 1 to 100 .

Fig. 2. Simulation for evaluating the quality of the parameter estimation using simultaneous state and parameter estimation by means Gaussian estimator. The mean of RMSE $\hat{e}_{k}^{\theta}$ (solid line) and the error variance $C_{k}^{\theta}$ (dashed line) for the estimated parameters are computed over 50 simulations with random selection of start values. After a certain transition time the Gaussian estimator offers an accurate estimation of both parameters.

where the weight $w_{s}$ is defined as $w_{s}=1 / D$. The vectors $\mu_{i}^{z}, \mathbf{C}_{i}^{z, z}$ are the components of the predicted joint density approximated with a Gaussian mixture

$$
\tilde{f}(z)=\sum_{i=1}^{L} w \cdot \mathcal{N}\left(\underline{z}-\underline{\mu}_{i}^{z}, \mathbf{C}_{i}^{z, z}\right)
$$

with mean and covariance [12]

$$
\begin{aligned}
& \underline{\mu}_{i}^{z}=\underline{g}\left(\underline{\mu}_{i}^{n}\right)+\mathbf{H}\left(\underline{\mu}_{i}^{n}\right) \cdot \underline{\mu}\left(\mu_{i}^{n}\right), \\
& \mathbf{C}_{i}^{z, z}=\mathbf{H}\left(\underline{\mu}_{i}^{n}\right) \cdot \mathbf{C}^{l \mid n} \cdot\left(\mathbf{H}\left(\underline{\mu}_{i}^{n}\right)\right)^{\mathrm{T}} .
\end{aligned}
$$

The matrix $\mathbf{C}^{l \mid n}$ represents the second moment of the conditional density

$$
f\left(\underline{x}^{l} \mid \underline{x}^{n}\right)=\mathcal{N}\left(\underline{x}^{l}-\underline{\mu}\left(\underline{x}^{n}\right), \mathbf{C}^{l \mid n}\right)
$$

with mean and covariance

$$
\begin{aligned}
& \underline{\mu}\left(\underline{x}^{n}\right)=\underline{\mu}^{l}+\mathbf{C}^{l, n} \cdot\left(\mathbf{C}^{n, n}\right)^{-1} \cdot\left(\underline{x}^{n}-\underline{\mu}^{n}\right), \\
& \mathbf{C}^{l \mid n}=\mathbf{C}^{l, l}-\mathbf{C}^{l, n}\left(\mathbf{C}^{n, n}\right)^{-1} \cdot \mathbf{C}^{n, l} .
\end{aligned}
$$

\section{B. Filtering}

The filter step or measurement update fuses the predicted state estimate with the measurement information. Since the measurement equation is linear, we apply the Kalman filter step [13] according to

$$
\underline{\mu}_{k}^{e}=\underline{\mu}_{k}^{p}+\mathbf{K}_{k}\left(\underline{\hat{y}}_{k}-\mathbf{H}_{k} \underline{\mu}_{k}^{p}\right), \mathbf{C}_{k}^{e}=\left(\mathbf{I}-\mathbf{H}_{k} \mathbf{K}_{k}\right) \mathbf{C}_{k}^{p},
$$

where

$$
\mathbf{K}_{k}=\mathbf{C}_{k}^{p} \mathbf{H}_{k}^{\mathrm{T}}\left(\mathbf{H}_{k} \mathbf{C}_{k}^{p} \mathbf{H}_{k}^{\mathrm{T}}+\mathbf{C}_{k}^{v}\right)^{-1}
$$

Here, $\mathbf{I}$ is an identity matrix and $\mathbf{C}_{k}^{v}$ is the covariance matrix of the measurement noise.

\section{Physical Constraints}

For ensuring the physical meaning of the model parameters, the part of a probability distribution function of the parameter estimates $\underline{\boldsymbol{x}}^{n} \sim \mathcal{N}\left(\underline{\mu}^{n}, \mathbf{C}^{n, n}\right)$ that violates the physical constraints $f\left(\underline{\boldsymbol{x}}^{n} \mid\left\{\underline{a} \leq \underline{\boldsymbol{x}}^{n} \leq \underline{b}\right\}\right)$ is discarded. The vectors $\underline{a}$ and $\underline{b}$ define the bounds of the physical parameters. The probability density of the state $\underline{\boldsymbol{z}}_{k}$ is given by a truncated Gaussian distribution

$$
f^{*}\left(\underline{x}^{n}, \underline{x}^{l}\right)= \begin{cases}d \cdot f\left(\underline{x}^{n}, \underline{x}^{l}\right) & \text { if } \underline{a} \leq \underline{\boldsymbol{x}}^{n} \leq \underline{b} \\ 0 & \text { elsewhen },\end{cases}
$$

where $f\left(\underline{x}^{n}, \underline{x}^{l}\right)$ stays for the joint density of the linear and nonlinear part of the state. The normalization factor $d$ is defined as

$$
d=\int_{-\infty}^{+\infty} \int_{\underline{a}}^{\underline{b}} f\left(\underline{x}^{n}, \underline{x}^{l}\right) \mathrm{d} \underline{x}^{n} \mathrm{~d} \underline{x}^{l} .
$$

The first two moments of the $f^{*}\left(\underline{x}^{n}, \underline{x}^{l}\right)$ can be computed analytically according to [13].

For enforcing the physical constraints after the prediction step, the joint density $f\left(\underline{x}^{n}, \underline{x}^{l}\right)=f^{p}\left(\underline{x}^{n}, \underline{x}^{l}\right)$ is represented by the predicted joint density. After the filter step, the updated joint density $f\left(\underline{x}^{n}, \underline{x}^{l}\right)=f^{e}\left(\underline{x}^{n}, \underline{x}^{l}\right)$ is truncated.

\section{EVAluATion}

The performance of the proposed approach is evaluated in simulations and an experiment on a pressure-regulated artificial heart. At first, the convergence of the estimated parameters to the true values is confirmed in simulations. Then, after describing the experimental setup, we analyze the proposed simultaneous state and parameter identification approach by evaluating the accuracy of physics-based tracking. By comparing the predicted results with highly accurate measurements, we check the accuracy of the obtained prediction.

\section{A. Simulation}

In the simulation, we consider the three-dimensional physicsbased model of the heart surface motion proposed in [4]. The linear part of the system state $\underline{x}^{l} \in \mathbb{R}^{168}$ describes the heart kinematics, such as the three-dimensional position and the velocity of the heart surface. The nonlinear part $\underline{\boldsymbol{x}}^{n} \in \mathbb{R}^{5}$ contains unknown model parameters $\underline{\boldsymbol{\theta}}_{k}=\left[\boldsymbol{\rho}_{k}, \boldsymbol{\alpha}_{1 k}, \boldsymbol{\alpha}_{2 k}, \boldsymbol{E}_{k}, \boldsymbol{\nu}_{k}\right]^{\mathrm{T}}$ given by equation (3). Further, we assume the material density $\rho_{k}$ of the heart, the damping coefficients $\boldsymbol{\alpha}_{1 k}$ and $\boldsymbol{\alpha}_{2 k}$, the Young's modulus 


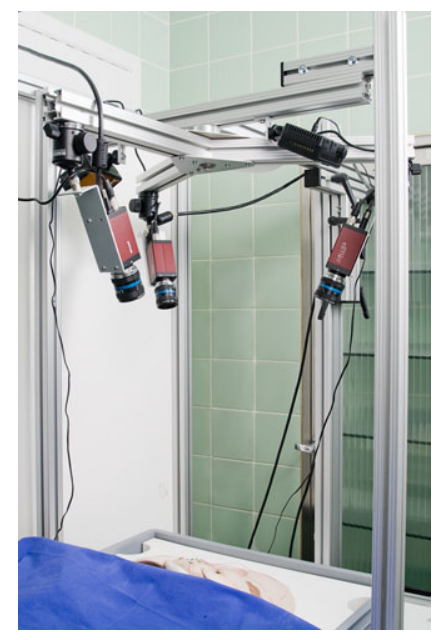

Fig. 3. Experimental setup for the evaluation of the physics-based tracking approach in an experiment with an artificial heart phantom.

$\boldsymbol{E}_{k}$ and the Poisson's ratio $\boldsymbol{\nu}_{k}$ as random variables. The true parameters of the monitored physical system are defined as $\rho=0.052 \mathrm{kgm}^{-3}, \alpha_{1}=6.096 \mathrm{Ns}^{2} \mathrm{~m}^{-1}$, $\alpha_{2}=0.001 \mathrm{Ns}^{2} \mathrm{~m}^{-1}, E=10000 \mathrm{MPa}, \nu=0.0407$. The system noise of the linear part of the state is assumed with the covariance $\mathbf{C}_{k}^{x^{l}}=\operatorname{diag}\{0.01, \ldots, 0.01\} \mathrm{mm}^{2}$. The system noise of the parameters is given by $\mathbf{C}_{k}^{x^{n}}=$ $\operatorname{diag}\{0.0004,0.0025,0.0025,10000,0.0001\}, \quad\left[\mathbf{C}_{k}^{x^{n}}\right]=$ $\operatorname{diag}\left\{\mathrm{kg}^{2} \mu \mathrm{m},\left(\mathrm{N}^{2} \mathrm{~s}^{2} \mathrm{~m}^{-1}\right)^{2},\left(\mathrm{Ns}^{2} \mathrm{~m}^{-1}\right)^{2}, \mathrm{MPa}^{2}, 1\right\}$.

The measurement noise variance is $\mathbf{C}_{k}^{v}=$ $\operatorname{diag}\{0.02, \ldots, 0.02\} \mathrm{mm}^{2}$.

The estimation quality of the GE is evaluated in $n=50$ Monte Carlo runs, where the initial estimates of the parameters are randomly chosen in every run. The value of every parameter is drawn from the normal distribution, which is defined by the mean corresponding to the true parameter value. The covariances of these distributions are $\mathbf{C}_{e}^{n, n}=$ $\operatorname{diag}\{0.09,0.001,0.001,1000000,0.0001\},\left[\mathbf{C}_{k}^{n, n}\right]=$ $\operatorname{diag}\left\{\mathrm{kg}^{2} \mu \mathrm{m},\left(\mathrm{Ns}^{2} \mathrm{~m}^{-1}\right)^{2},\left(\mathrm{Ns}^{2} \mathrm{~m}^{-1}\right)^{2}, \mathrm{MPa}^{2}, 1\right\}$.

The root-mean-square error (RMSE) is computed over all Monte Carlo runs $n$ and is defined by mean and variance

$\hat{e}_{k}^{\theta}=\frac{1}{n} \sum_{r=1}^{n}\left\|\hat{\theta}_{k, r}^{e}-\underline{\theta}_{\text {true }}\right\|, C_{k}^{\theta}=\frac{1}{n-1} \sum_{r=1}^{n}\left(\underline{e}_{k, r}^{\theta}-\hat{e}_{k, r}^{\theta}\right)^{2}$

for every time step $k$. The results of the simulation are shown in Fig. 2. It is obvious that the parameters estimated by GE converge to the true parameter values after a certain transition time. Therefore, the proposed estimator successfully detects the uncertain parameters.

\section{B. Experiment}

The aim of the experiment is the evaluation of the prediction quality of physics-based tracking. Since the parameters of the real system are unknown, the accuracy of the prediction is evaluated in comparison to the measurements.

\section{1) Experimental Setup:}

Physics-based tracking of the heart surface motion is evaluated by measuring the motion of the pressureregulated artificial heart. Its $3 \mathrm{D}$ motion is reconstructed based on three camera views provided by a trinocular camera system shown in Fig. 3. The cameras are placed at a distance of $0.5 \mathrm{~m}$ from one another. Their focal length is about $35 \mathrm{~mm}$ and the field of view is $12.8 \times 17.0 \mathrm{~cm}$. The high resolution of $1920 \times 1080$ pixel allows an accurate $3 \mathrm{D}$ reconstruction. The covariance of the measurement noise is $\mathbf{C}_{k}^{v}=\operatorname{diag}\{0.01, \ldots, 0.01\} \mathrm{mm}^{2}$ in all three directions. The motion of the artificial heart is approximated by the physics-based model proposed in [4]. The initial values of the unknown physical parameters $\underline{\boldsymbol{\theta}}=\left[\rho, \alpha_{1}, \alpha_{2}, E, \nu\right]^{\mathrm{T}}$ are determined by the weighted least-squares calibration procedure, proposed in Section IV. The initial estimates of the physical parameters are assumed as $\underline{\mu}_{e}^{n}=[1291,0.55,0.02,24,0.33]^{\mathrm{T}}$, $\left[\mu_{e}^{n}\right]=\left[\mathrm{kgm}^{-3}, \mathrm{Ns}^{2} \mathrm{~m}^{-1}, \mathrm{Ns}^{2} \mathrm{~m}^{-1}, \mathrm{MPa}, 1\right]$. The process noise of the linear part of the state describing the heart kinematics is assumed with the covariance $\quad \mathbf{C}_{k}^{x^{l}}=\operatorname{diag}\{0.012, \ldots, 0.012\} \mathrm{mm}^{2}$. The system noise of the parameters is given by $\mathbf{C}_{k}^{x^{n}}=\operatorname{diag}\{100,0.0025,0.0025,1,0.0001\},\left[\mathbf{C}_{k}^{x^{n}}\right]=$ diag $\left\{\mathrm{kg}^{2} \mu \mathrm{m},\left(\mathrm{Ns}^{2} \mathrm{~m}^{-1}\right)^{2},\left(\mathrm{Ns}^{2} \mathrm{~m}^{-1}\right)^{2}, \mathrm{MPa}^{2}, 1\right\}$. The lower bound $\underline{a}=[0.5,0,0,0.08,0]^{\mathrm{T}}$ and upper bound $\underline{b}=[20000,1,1,26,0.5]^{\mathrm{T}}$ for estimating the parameter values by means of truncation (7) are derived according to information provided by a manufacturer of the artificial heart. The a-priori known physical constraints are also considered. Then, the state vector $\underline{\boldsymbol{x}}_{k}^{l} \in \mathbb{R}^{168}$ that describes the displacement and velocity of the heart surface and the physical parameters $\underline{\boldsymbol{x}}_{k}^{n} \in \mathbb{R}^{5}$ are estimated simultaneously by GE. The input vector is determined by the atrial pressure, which can be monitored in cardiac surgery by a central line catheter [14]. In the test environment, the pressure inside the artificial heart is measured by a pressure sensor. For evaluation of the prediction quality of the proposed physics-based tracking approach, we consider the prediction error, whose mean and covariance are defined as an absolute deviation of the predicted state $\underline{\hat{z}}_{k}^{p}$ from the observed measurements of the heart surface position $\underline{\hat{y}}_{k} \in \mathbb{R}^{m}$. The dimensionality of the measured state is defined by $m=42$. This way, we check if the accuracy of the proposed system model is comparable with the measurement accuracy.

2) Experimental Results: The estimated physical parameters converge to the vector $\underline{\hat{\theta}}=[1306,0.554,0.59,24.95,0.32]^{\mathrm{T}}, \quad[\underline{\hat{\theta}}]=$ $\left[\mathrm{kgm}^{-3}, \mathrm{Ns}^{2} \mathrm{~m}^{-1}, \mathrm{Ns}^{2} \mathrm{~m}^{-1}, \mathrm{MPa}, 1\right]$ with uncertainties $\mathbf{C}^{\theta}=\operatorname{diag}\{0.2,0.006,0.006,0.63,0.0019\}, \quad\left[\mathbf{C}^{\theta}\right]=$ $\operatorname{diag}\left\{\mathrm{kg}^{2} \mu \mathrm{m},\left(\mathrm{Ns}^{2} \mathrm{~m}^{-1}\right)^{2},\left(\mathrm{Ns}^{2} \mathrm{~m}^{-1}\right)^{2}, \mathrm{MPa}^{2}, 1\right\}$.

In Fig. 4, the state prediction error computed over $m=42$ observed states is visualized at every time step. In order to emphasize the high quality of simultaneous state and parameter estimation, we compare the results provided by the Kalman filter assuming that the parameters of the model are exactly detected by the calibration procedure. For GE, the mean of the state prediction error computed over all time steps is about $0.041 \mathrm{~mm}$, whereas the Kalman Filter produces the error of about $0.0823 \mathrm{~mm}$. Therefore, the performance of the physics-based tracking is improved 


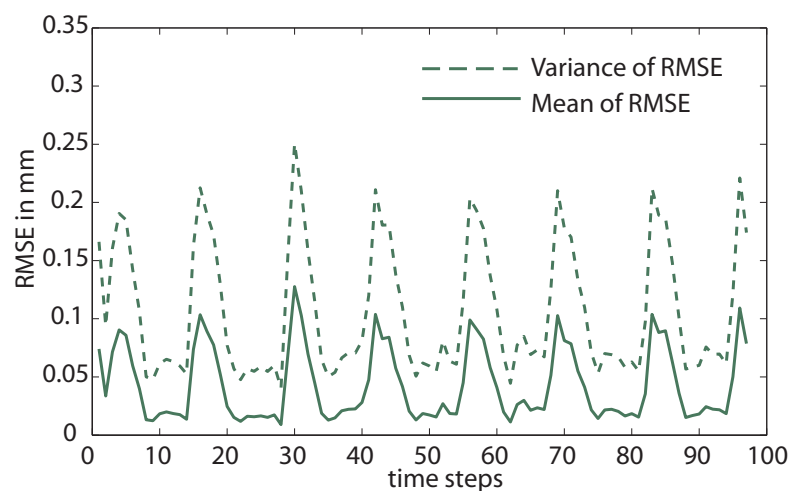

(a) State prediction error in comparison to the measurements for time steps 1 to 100 provided by simultaneous state and parameter estimation with the Gaussian estimator.

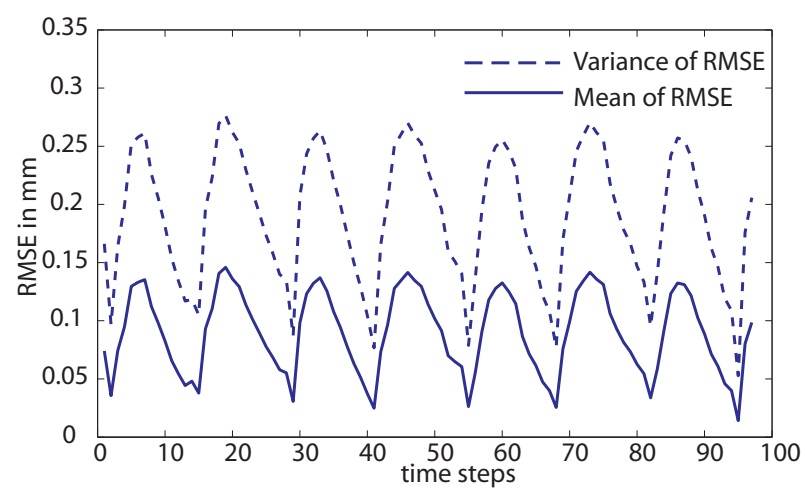

(b) State prediction error in comparison to the measurements for time steps 1 to 100 provided by the Kalman filter assuming the calibrated parameters as exactly known.

Fig. 4. Evaluation of the prediction quality of the physics-based tracking in experiment on a artificial pressure-regulated heart. The mean $\hat{e}_{k}^{z}$ and variance $C_{k}^{z}$ of the RMSE is computed over the number of observations. The simultaneous state and parameter estimation by means the Gaussian estimator is more accurate than the results provided by Kalman filter.

by the simultaneous state and parameter estimation using GE. Accuracy may be further increased by obtaining a more realistic physical model of the artificial heart motion.

The computation time for the processing of a highdimensional nonlinear estimation problem containing 175 state variables is on average $374 \mathrm{~ms}$. The Kalman filter needs $105 \mathrm{~ms}$ for processing of a linear containing 170 state variables. The efficiency of the nonlinear estimation can be increased by a parallel computing.

\section{CONCLUSIONS}

In this paper, a physics-based probabilistic approach for tracking the heart surface motion is presented. It aims to be applied in beating heart surgery. In contrast to existing approaches, the physical background information about complex heart dynamics and material properties is considered in the prediction of the heart behavior. Furthermore, the uncertainties of the system dynamics, model parameters, and measurements are handled in a systematic way by exploiting simultaneous state and parameter estimation. Moreover, the efficient solution of the estimation problem is proposed by decomposing the state into linear and nonlinear substructures. The experimental results are very promising, and by further parallelization, real-time processing can be achieved.

The solution of the parameter identification problem allows for a personalized physical model that can be calibrated during an operation on a beating heart. As a consequence, this opens up possibilities to apply probabilistic physicsbased tracking of the heart surface motion in a clinical environment.

\section{REFERENCES}

[1] T. Ortmaier, M. Groeger, D. H. Boehm, V. Falk, and G. Hirzinger, "Motion Estimation in Beating Heart Surgery," IEEE Transactions on Biomedical Engineering, vol. 52, no. 10, pp. 1729-1740, Oct. 2005.

[2] D. Stoyanov, G. P. Mylonas, F. Deligianni, A. Darzi, and G. Z. Yang, "Soft-Tissue Motion Tracking and Structure Estimation for Robotic Assisted MIS Procedures," in Proceedings of the Medical Image Computing and Computer Assisted Interventions (MICCAI 2005), vol. 2, 2005, pp. 139-146.
[3] T. Bader, A. Wiedemann, K. Roberts, and U. D. Hanebeck, "Modelbased Motion Estimation of Elastic Surfaces for Minimally Invasive Cardiac Surgery," in Proceedings of the 2007 IEEE International Conference on Robotics and Automation (ICRA 2007), Rome, Italy, Apr. 2007, pp. 2261-2266.

[4] E. Bogatyrenko, U. D. Hanebeck, and G. Szabo, "Heart Surface Motion Estimation Framework for Robotic Surgery Employing Meshless Methods," in Proceedings of the 2009 IEEE/RSJ International Conference on Intelligent Robots and Systems (IROS 2009), October 2009.

[5] J. R. Huddle and D. A. Wismer, "Degradation of Linear Filter Performance Due to Modeling Error," IEEE Transactions Automatic Control, vol. 13, no. 4, pp. 421-423, 1968.

[6] M. Sermesant, P. Moireau, O. Camara, J. Sainte-Marie, R. Andriantsimiavona, R. Cimrman, D. Hill, D. Chapelle, and R. Razavi, "Cardiac Function Estimation from MRI using a Heart Model and Data Assimilation: Advances and Difficulties," in Functional Imaging and Modeling of the Heart, vol. 10, 2006, pp. 642-656.

[7] P. Shi and H. Liu, "Stochastic Finite Element Framework for Cardiac Kinematics Function and Material Property Analysis," in Proceedings of the Medical Image Computing and Computer-Assisted Intervention (MICCAI 2002), 2002, pp. 634-641.

[8] F. Sawo, V. Klumpp, and U. D. Hanebeck, "Simultaneous State and Parameter Estimation of Distributed-Parameter Physical Systems based on Sliced Gaussian Mixture Filter," in Proceedings of the 11th International Conference on Information Fusion (Fusion 2008), Cologne, Germany, Jul. 2008, pp. 1-8.

[9] D. Uciński, Optimal Measurement Methods for Distributed Parameter System Identification, 2005.

[10] C. P. Robert and G. Casella, Monte Carlo Statistical Methods. Springer Science and Business Media Inc., 2004.

[11] M. F. Huber and U. D. Hanebeck, "Gaussian Filter based on Deterministic Sampling for High Quality Nonlinear Estimation," in Proceedings of the 17th IFAC World Congress (IFAC 2008), vol. 17, no. 2, Seoul, Korea, Jul. 2008

[12] F. Beutler, M. F. Huber, and U. D. Hanebeck, "Gaussian Filtering using State Decomposition Methods," in Proceedings of the 12th International Conference on Information Fusion (Fusion 2009), Seattle, Washington, July 2009.

[13] D. Simon, Optimal State Estimation: Kalman, H Infinity, and Nonlinear Approaches. Wiley-Interscience, 2006.

[14] R. Zahorec and M. Holoman, "Transatrial Access for Left Atrial Pressure Monitoring in Cardiac Surgery Patients," European Journal of Cardio-Thoracic Surgery, vol. 11, pp. 379-380, 1997. 